\title{
A REVIEW STUDY ON ORTHOGONAL FREQUENCY DIVISION MULTIPLEXING
}

\section{KEY WORDS: OFDM}

(Orthogonal Frequency Division Multiplexing), Multiplexer, intersymbol interference (ISI)

\section{Vishal Ghode* Suresh Gawande Satyarth Tiwari \\ Bhabha University, Bhopal. *Corresponding Author \\ Bhabha University, Bhopal. \\ Bhabha University, Bhopal.}

The advancement in the up-grading of technology and proliferation in data accessibility is changing the economics of digital communication; therefore, this enables the reexamining of the previous design and approach to network architectures. This research paper focused on advancements in technology in the past few years and concentrate on OFDM (Orthogonal Frequency Division Multiplexing).

\section{INTRODUCTION}

In digital communication, the modulation of data is perform on one supporting frequency. The bandwidth is employ to each symbol. Such a system lead to Inter-Symbol Interference (ISI) while in case of the selective channel. The OFDM (Orthogonal frequency division multiplexing) idea is to differentiate the available spectrum into various Orthogonal Sub-Channel. It is do to achieve flat fading. OFDM is the best modulation technique in wireless communication to operate huge date with robustness and improvement in radio channel impairments. Many research organization in the world have specialized teams working in the optimization of OFDM systems. In an OFDM scheme, many orthogonal, overlapping, narrow-band sub-carriers are transmitte in parallel. These carriers divide the available transmission bandwidth. The separation of subcarriers is in such a way that there is a very compact spectral application. OFDM provides access to have overlapping sub-Channels in the frequency domain, hence boost the transmission rate. The OFDM is prompted mainly because of the accessibility of multi-path interference at the receiver end. Multi-path phenomenon generates two effects

(a) Frequency selective fading and

(b) Inter-symbol interference (ISI).

\section{Transmitter}

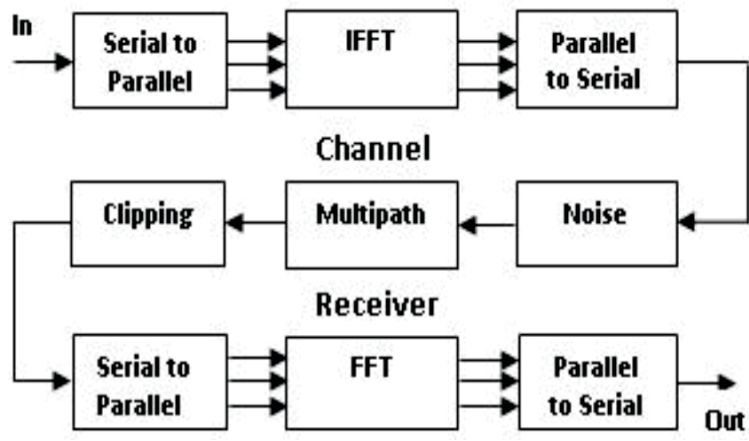

Fig 4.8: OFDM model

\section{LITERATURE REVIEW}

Yu He \& et al. [1] performed experimental work on silicon photonic chip in sub-wave grating (SWG) multiplexing to improve optical transmission at a higher order. By single polarization at 11 Mod multiplexers in which the desired results at $1545 \mathrm{~nm}, 11$ channels have crosstalk between $15.4 \mathrm{~dB}$ to $-26.4 \mathrm{~dB}$, and low insertion loss of $0.1 \mathrm{~dB} 2.6 \mathrm{~dB}$ respectively. The useful refractive index (SWG) at $1545 \mathrm{~nm}$ with a variation of $\pm 20 \mathrm{~nm}$ has a crosstalk value of $10.4 \mathrm{~dB}$ and an insertion value below $4.0 \mathrm{~dB}$. They suggested having a structural analysis to improve the efficiency of various supporting parameters. The second concern about the research is that it will increase the transmission efficiency using PDM to achieve a higher-order state.
D. Gonzalez-Andrade and et al. [2] propose current bandwidth capacity provided by wavelength division multiplexing and polarization division multiplexing is insufficient to keep up with the increasing bandwidth demand required for new services. Full $3 \mathrm{D}$ simulations of the designed mode converter and de/multiplexer show insertion losses less than $0.84 \mathrm{~dB}$ and crosstalk less than -20 degree over an unprecedented bandwidth of $300 \mathrm{~nm}(1.4(1.4-1.7 \mu \mathrm{m})$. The total footprint of the suggested device is only $36 \mu \mathrm{m} \times 3.7$ $\mu \mathrm{m}$. Under his work, an ultra-broadband two-mode converter and de/multiplexer comprised of an SWG-MMI, a $\frac{\mu}{2}$ phase shifter and symmetric $y$-junction is proposed for the first time. Tolerance study shows good fabrication tolerance to errors between $-20 \mathrm{~nm}$ and $+20 \mathrm{~nm}$ for the entire device. The results presented in this paper lead the new dimension for compact mode division multiplexing device that can be used together with wavelength- division multiplexing to further increase bandwidth capacity in Silicon photonic interconnects.

Daoxin Dai [3] proposed that various photonic integrated devices have been developed successfully with silicon-oninsulator (SOI) nanowires in the past decade. It is well known, that SOI - nanowire waveguides have Ultrahigh index contrast $(\Delta)$ and ultrahigh birefringence. As a result, the structures and the design rules of a silicon photonic device are probably very different from those of the conventional case of low $\Delta$ optical waveguides. Silicon photonic Integrated circuits with these building blocks are also reviewed, including hybrid (de) multiplexers and reconfigurable optical add-drop multiplexers. His paper provides a review of recent progress on advance passive Silicon photonic devices with asymmetric waveguide structures.

George Kalfas, \& et al. [4] proposed architecture leverage optical transceiver, optical add/drop multiplexer and optical beam farming integrated photonics towards a digital signal processing analogue front haul. The front haul infrastructure's functional administration is achieving utilizing a Packetized Medium Transparent Dynamic Bandwidth Allocation Protocol. Preliminary results show that the protocol can facilitate $\mathrm{Gb} / \mathrm{s}$ - enabled data transport while abiding by the 5G low KPIs in various network traffic conditions. This article has presented a novel FiWi, C-RAN capable of optimally meeting the needs of mmWave urban- area and hotspot scenarios. His proposal relies on cost-effective a-RoFcapable devices, including EMLS, OBFNs and ROADMs. The converged topical/wireless resource allocation is achieve through MT-DBA protocol. Result concludes the future possibility of $5 \mathrm{Gmm}$ wave network via a medium transparent protocol.

Luhua Xu \& et al. [5] experimentally demonstrate a compact broadband "polarization beam splitter (PBS) based on a multimode interference (MMI) coupler with internal photonic 
crystal (PC) for the silicon-on-insulator" plate form. A wide range of study on device operation, including photonic band gap and impact on the digital structure internal communication on MMI coupler on each mode. The experimental device achieves measured extinction ratios higher than $20 \mathrm{~dB}$ and insertion losses lower than $2 \mathrm{~dB}$ for both polarization over a $77 \mathrm{~nm}$ wavelength range from 1522 to 1599 nm covering the entire C-band with a device length of only $71.5 \mu \mathrm{m}$. His team experimentally demonstrated a compact and broadband PBS based on the MMI coupler with the SOI platform's internal PC. The designed PC -MMI PBS has been fabricated using electron beam lithography and the feature size used in our design in CMOS compatible.

Shibsankar Das, \& et al. [6] complementary sets of sequence (CSS) and complete complementary codes (CCC) have found numerous applications in wireless communication and Radar sensing owing to their perfect periodic correlation properties. Proposed a multiplier free implementation of this generator based on Multiplexer and read-only memories (ROMs). A new recursive algorithm based on PU Matrix has been developing to generate polyphase CSS of $\mathrm{M}$ sequences with length $\mathrm{M}^{N}$ for arbitrary $\mathrm{M}>1$. Proposed algorithm can generate polyphase CCC. Furthermost he propose a more computationally efficient PU generator based on multiplexer and ROMs. Therfore, it can be used as an advantage for applications using sequences for coding (e.g. coded-keying OFDM) or to select lines with good correlation or spectral properties among a large number of candidates.

Weijie Chang \& et al. [7] propose and experimentally demonstrate a novel ultra-compact multimode waveguide crossing our proposed device occupied only a compact footprint of $34 \times 34 \mu \mathrm{m}^{2}$.The measured results indicates indicates our fabricated device exhibited a high performance with the insertion loss of less than $0.9 \mathrm{~dB}$, crosstalk lower than $-24 \mathrm{~dB}$ from 1.52 to $1.60 \mu \mathrm{m}$ for all the three modes. In summary, he proposed and experimentally demonstrated and ultra-compact multimode waveguide crossing, which could implement three modes simultaneously our proposed scheme could be easily expande to implements more methods. Furthermore, converting a higher-order way to the fundamental mode may offer an attractive approach to simultaneously implementing on-chip multimode signal processing.

Cyriel Minkenberg, \& et al. [8] proposed that silicon photonic chips change the economics of digital communication. Networks architects should be able to select topologies based on their merits and not merely from tradition. Present the design and implementation of Topanga pocket switch optoASIC with the requisite technologies to free data centre topologies from controls induced by some electrical scope degree. His experiment on integrating silicon photonics inside the package, Topanga provides optical I/O with lower energy per bit at a lower cost per port than existing solutions. He has demonstrated package optics' feasibility based on our low-cost, low power Silicon photonics platform by building a prototype implementation, the Topanga optoASIC and a complete reference system. They showed how this technology improves energy efficiency and reduces the cost per bandwidth compared to conventional switch ASICs, with modular transceiver optics. Providing basses for integrating Silicon photonics inside the package combines the low cost and low power dissipation of shortrange electrical interconnects with the long reach of singlemode optics. It enables a fundamental rethinking of data centre network architecture.

Hasan Hosseinzadeh, \& et al. [9] focused on challenges in CMOS circuits, one of the most important of the features' size. Alternative technologies, such as quantum-dot cellular automata (QCA) are incipient to resolve this issue. In QCA, the scale of the main structures is tiny, which can cause faults. In this paper, they proposed a new fault-tolerant mainstream gate. They investigate the suggested system's performance when common faults occur and then compare the previous structures' results. Using this structure, we propose a new design for a fault-tolerant full-adder. QCA Designer is used to implementing and putting on suggested systems. In this proper presented a novel fault tolerance majority gate. The physical investigation confirmed the simulation results. The results show that the proposed design, which provides $100 \%$ fault-tolerance under one missing cell defect. It also provides $54.5 \%$ fault tolerance under the two absent cells defect.

Seyed-Sajad Ahmadpour \& et al. [10] propose Quantum-dot cellular automaton ( $\mathrm{QCA}$ ) has emerged as one of the best alternatives to CMOS technology in Nanoscale. The use of multiplexer circuits in QCA technology has been of boundless interest to researchers due to its extensive use in memory circuits and ALUs. In this paper, a novel fault-tolerant there input majority gate is initially suggested. The suggested structure has been investigating against all kinds of cell omission, extra cell deposition and cell displacement defects. Finally using the proposed system, a novel single-layer 2:1 multiplexer is present. The results of comparisons indicate that the proposed designs are more reliable than the existing techniques. Furthermore, QCAPro power estimator tool is employed to estimate the energy dissipation of the proposed structure. The outcomes of the comparisons showed that the proposed fault-tolerant multiplexer has a higher degree of fault tolerance than other designs.

Firdous Ahmad \& et al. [11] propose Quantum-dot Cellular Automata (QCA) is radical nanotechnology, which works at Nanoscale. In this paper an optimal design of $2^{n}: 1$ Multiplexer (MUX) and 1:2 $2^{n}$ Demultiplexer (DeMUX) represented. New execution and simulation results of the proposed $4^{n}: 1$ MUX and $1: 4^{n}$ DeMUX is developed using the propose 2:1 MUX. Also, a novel concept of QCA based Multiplexing/ Demultiplexing is presente. It paves a new way of sharing a single communication link among the number of devices at nano-regime. The proposed designs have shown significant improvements compared to the previously designed multiplexers in terms of area and complexity.

Abbas Vosoogh \& et al. [12] propose this paper presents a diplexer's integration with a corporate feed network of a high gain slot array antenna at the Ka-band. The proposed integrated diplexer-antenna module consists of three distinct metal layers without electrical contacts between the different layers based on the recently introduced gap waveguide technology. The designed module has two channels of 650$\mathrm{MHz}$ bandwidths each with centre frequency 28.21 and 29.21 $\mathrm{GHz}$. The fabricated prototype provides significant radiation and input impedance characteristics. The measured input reflection coefficient for both $\mathrm{Tx} / \mathrm{Rx}$ ports is better than $-13 \mathrm{~dB}$. The measured antenna efficiency is better than $60 \%$ in the design Passband, including the diplexer's losses.

Rubana B. Priti \& et al. [13] proposed experimentally demonstrating a scalable and broadband reconfigurable multimode de-multiplexer/switch (RMDS) mode-divisions multiplexed inter-and intra-chip data communication system. The RMDS uses a thermo-optically tuned tapered multimode interference coupler, allowing concurrent transmission of the essential and first-order quasi transverse electric modes in Cband. It is used as the building block in a mode demultiplexer with $-20 \mathrm{~dB}$ crosstalk which reconfigured as a mode selecting switch with $10.9 \mu$ s switching time. This is the first demonstrated of such a device that can be reconfigured to perform both mode de-multiplexing and mode switching of high speed optical data. The RMDS can be used as a building block to deploy high capacity optical links in shortreach interconnects allowing the parallel transmission and switching of multiple independent data packets for a potential increase in link energy per bit performance. 


\section{Theory:}

Multimedia is efficiently an infrastructure technology with broadly different roots in telecommunications, entertainment, computing and printing. Novel applications are emergent, not just in the wired atmosphere, but also in the mobile one. Currently, only low bit-rate data services are accessible to cell phone users. The radio environment is challenging due to the numerous reflected waves and different effects. Adaptive Equalization method at receiver end will be a solution. But may have practical barriers while operating equalization in real-time at $\mathrm{Mb} / \mathrm{s}$ with small size and reduced hardware cost. The OFDM is suitable to eliminate complex equalizers.OFDM is robust in adversative channel conditions and allows a high level of spectral efficiency. It effectively mitigates performance degradations due to multi-path have the potential of deep fades in some portion of the spectrum.

The OFDM can operate considerable delay spreads easier due to the carriers' liberation and the flexibility of changing the cyclic introduce length. Numerous techniques schemes allow many cell phone users to share a limited amount of radio spectrum simultaneously. The sharing is essential to achieve high capacity by allocating the available bandwidth (or the available amount of channels) to multiple users. For quality communications, this must be done without severe degradation in the performance of the system. FDMA, TDMA and CDMA are the well-known multiplexing methods used in wireless communication systems. While employed with the wireless networks using these techniques various problems encountered are:-

(1) Multi-path fading

(2) Time dispersion which leads to inter-symbol interference (ISI)

(3) Lower bit rate capacity

(4) Prerequisite of larger transmit power for high bit rate and

(5) Less spectral efficiency.

A disadvantage of the FDMA technique is its Bad Spectrum Usage. Disadvantages of the TDMA technique is Multi-path Delay spread problem. In distinctive terrestrial broadcasting, the transmitted signal reaches at the receiver using different paths of dissimilar lengths. Since multiple kinds of signals interfere with each other, it becomes difficult to extract the original information. These orthogonal frequency division multiplexing (OFDM) techniques provide a better solution for the problems mentioned above.

\section{CONCLUSIONS}

The OFDM makes resourceful use of existing spectrum by letting overlapping between the carriers. It mainly converts the high data rate stream into numerous parallel lower data rate streams, diminishing the frequency of selective diminishing. It is computationally efficient due to the use of FFT techniques to implement modulation and demodulation functions.

From the model results, it is experimental that the BPSK allows the BER to be enhanced in a noisy channel at the sake of extreme data transmission capacity. Use of QPSK allows higher transmission capacity, but at the cost of a slight increase in the error probability. It is because of QPSK uses two bits per symbol. Hence QPSK is easily affected by the noise. Therefore OFDM with QPSK requires larger transmit power. From the results, the use of OFDM with QPSK is beneficial for short-distance transmission link, whereas for long-distance transmission link OFDM with BPSK will be preferable.

\section{SCOPE OF FUTUREWORK}

1) An motivating topic for future research is to perform more extensive performance comparisons between FFT based OFDM, DHT based OFDM, and DCT found OFDM systems under additional real-world channel impairments, such as multi-path fading, time dispersion which leads to inter symbol interference (ISI).

2) The OFDM signal's main problems are susceptible to carrier frequency offset, and its high Peak to Average Power Ratio (PAPR). So, these three transform-based OFDM systems can be tested for these problems.

\section{REFERENCES:}

[1] Y.He et al.,"Silicon high-order mode (De)multiplexer on single polarization," J.Light.Technol.,vol.36, no. 24,pp. 5746-5753,2018.

[2] D. González-Andrade et al., "Ultra-Broadband Mode Converter and Multiplexer Based on Sub-Wavelength Structures," IEEE Photonics J., vol. 10, no.2,pp. 1-11,2018.

[3] D. Dai, "Advanced Passive Silicon Photonic Devices With Asymmetric Waveguide Structures," Proc.IEEE, vol. 106,no. 12,pp.2117-2143,2018.

[4] G. Kalfas et al., "Next Generation Fiber-Wireless Fronthaul for 5G mmWave Networks," IEEE Commun.Mag., vol.57,no.3,pp. 138-144,2019.

[5] L. Xu et al., "Compact Broadband Polarization Beam Splitter Based on Multimode Interference Coupler with Internal Photonic Crystal for the SOI Platform,"J.Light.Technol., vol.37, no.4,pp. 1231-1240,2019.

[6] S. Das, S. Budišin, S. Majhi, Z. Liu, and Y. L. Guan, "A Multiplier-Free Generator for Polyphase Complete Complementary Codes," IEEE Trans. Signal Process., vol. 66, no. 5, pp. 1184-1196,2018.

[7] W. Chang et al., "An Ultracompact Multimode Waveguide Crossing Based on Subwavelength Asymmetric Y-Junction," IEEE Photonics J., vol. 10, no. 4, pp. $1-8,2018$.

[8] C. Minkenberg et al., "Reimagining datacenter topologies with integrated silicon photonics,"J. Opt. Commun.Netw., vol. 10, no. 7, pp.B126-B139,2018.

[9] H. Hosseinzadeh and S. R. Heikalabad, "A novel fault tolerant majority gate in quantum-dot cellular automata to create a revolution in design of fault tolerant nanostructures, with physical verification," Microelectron. Eng., vol. 192 , pp. 52-60,2018.

[10] S. S. Ahmadpour and M. Mosleh, "A novel fault-tolerant multiplexer in quantum-dot cellular automata technology," J. Supercomput., vol. 74, no. 9, pp. $4696-4716,2018$.

[11] F. Ahmad, "An optimal design of QCA based 2n:1/1:2n multiplexer/ demultiplexer and its efficient digital logic realization," Microprocess. Microsyst., vol.56, pp.64-75,2018.

[12] A. Vosoogh, M. S. Sorkherizi, A. U. Zaman, J. Yang, and A. A. Kishk, "An Integrated Ka-Band Diplexer-Antenna Array Module Based on Gap Waveguide Technology with Simple Mechanical Assembly and No Electrical Contact Requirements," IEEE Trans. Microw. Theory Tech., vol. 66, no. 2, pp. 962-972,2018.

[13] R. B. Priti and O. Liboiron-Ladouceur, "A reconfigurable multimode demultiplexer/ switch for mode-multiplexed silicon photonics interconnects,"'IEEEJ. Sel.Top. Quantum Electron., vol. 24, no. 6,2018. 